\title{
Longitudinal relaxation time editing for acetylcarnitine detection with H-MRS
}

Citation for published version (APA):

Lindeboom, L., Bruls, Y. M. H., van Ewijk, P. A., Hesselink, M. K. C., Wildberger, J. E., Schrauwen, P., \& Schrauwen-Hinderling, V. B. (2017). Longitudinal relaxation time editing for acetylcarnitine detection with H-MRS. Magnetic Resonance in Medicine, 77(2), 505-510. https://doi.org/10.1002/mrm.26149

Document status and date:

Published: 01/02/2017

DOI:

10.1002/mrm.26149

Document Version:

Publisher's PDF, also known as Version of record

Document license:

Taverne

Please check the document version of this publication:

- A submitted manuscript is the version of the article upon submission and before peer-review. There can be important differences between the submitted version and the official published version of record.

People interested in the research are advised to contact the author for the final version of the publication, or visit the DOI to the publisher's website.

- The final author version and the galley proof are versions of the publication after peer review.

- The final published version features the final layout of the paper including the volume, issue and page numbers.

Link to publication

\footnotetext{
General rights rights.

- You may freely distribute the URL identifying the publication in the public portal. please follow below link for the End User Agreement:

www.umlib.nl/taverne-license

Take down policy

If you believe that this document breaches copyright please contact us at:

repository@maastrichtuniversity.nl

providing details and we will investigate your claim.
}

Copyright and moral rights for the publications made accessible in the public portal are retained by the authors and/or other copyright owners and it is a condition of accessing publications that users recognise and abide by the legal requirements associated with these

- Users may download and print one copy of any publication from the public portal for the purpose of private study or research.

- You may not further distribute the material or use it for any profit-making activity or commercial gain

If the publication is distributed under the terms of Article $25 \mathrm{fa}$ of the Dutch Copyright Act, indicated by the "Taverne" license above, 


\title{
Longitudinal Relaxation Time Editing for Acetylcarnitine Detection with ${ }^{1} \mathrm{H}-\mathrm{MRS}$
}

\author{
Lucas Lindeboom, ${ }^{1,2 *}$ Yvonne M.H. Bruls, ${ }^{1,2 \dagger}$ Petronella A. van Ewijk, ${ }^{1 \dagger}$ \\ Matthijs K.C. Hesselink, ${ }^{2}$ Joachim E. Wildberger, ${ }^{1}$ Patrick Schrauwen, ${ }^{2}$ and \\ Vera B. Schrauwen-Hinderling ${ }^{1,2}$
}

\begin{abstract}
Purpose: Acetylcarnitine formation is suggested to be crucial in sustaining metabolic flexibility and glucose homeostasis. Recently, we introduced a method to detect acetylcarnitine in vivo with long TE ${ }^{1} \mathrm{H}$-MRS. Differences in $\mathrm{T}_{1}$ relaxation time between lipids and acetylcarnitine can be exploited for additional lipid suppression in subjects with high myocellular lipid levels.

Methods: Acquisition of spectra with an inversion recovery sequence was alternated with standard signal acquisition to suppress short $T_{1}$ metabolite signals. A proof of principle experiment was conducted in a lean subject and the new approach was subsequently tested in four overweight/obese subjects.

Results: Using the new $T_{1}$ editing approach, lipid signals in spectra of skeletal muscle can be (additionally) suppressed by a factor of 10 using a TI of $900 \mathrm{~ms}$. Combination of the long TE protocol with the $T_{1}$ editing resulted in a well-resolved acetylcarnitine peak in the obese subjects.

Conclusion: The $T_{1}$ editing approach suppresses short $T_{1}$ metabolites and offers a new contrast in ${ }^{1} \mathrm{H}-\mathrm{MRS}$. The approach should be used in combination with a long TE in subjects with high lipid contamination for accurate quantification of the acetylcarnitine concentration. Magn Reson Med 77:505-510, 2017. (c) 2016 International Society for Magnetic Resonance in Medicine
\end{abstract}

Key words: acetylcarnitine; $\mathrm{T}_{1} ; \mathrm{T}_{2}$; inversion recovery; ${ }^{1} \mathrm{H}-$ MRS

\section{INTRODUCTION}

It has recently been proposed that the formation of acetylcarnitine is essential in maintaining metabolic flexibility and glucose homeostasis (1-3). While high levels of acetyl-CoA are known to inhibit pyruvate dehydrogenase (PDH) complex activity, acetylcarnitine can be formed from excess acetyl-CoA, in a reaction catalyzed by the

\footnotetext{
${ }^{1}$ Department of Radiology, NUTRIM school for Nutrition and Translational Research in Maastricht, Maastricht University Medical Center, Maastricht, The Netherlands.

${ }^{2}$ Department Human Biology and Human Movement Sciences, NUTRIM school for Nutrition and Translational Research in Maastricht, Maastricht University Medical Center, Maastricht, The Netherlands.

*Correspondence to: Lucas Lindeboom, Ph.D., Department of Radiology, Maastricht University Medical Center, PO Box 5800, 6202 AZ Maastricht. E-mail: lucas.lindeboom@maastrichtuniversity.nl

'Drs. Bruls and van Ewijk contributed equally to this work.

Received 23 September 2015; revised 11 January 2016; accepted 13 January 2016

DOI 10.1002/mrm.26149

Published online 17 February 2016 in Wiley Online Library (wileyonlinelibrary. com).
}

(C) 2016 International Society for Magnetic Resonance in Medicine enzyme carnitine acetyltransferase (CrAT). As such, the formation of acetylcarnitine can be viewed as a mitochondrial rescue mechanism to maintain low acetyl-CoA concentrations and to sustain aerobic pyruvate oxidation.

While research on acetylcarnitine has long been limited to biochemical analysis in muscle biopsies, it has been shown that exercise-induced acetylcarnitine production can be detected with short echo time (TE) proton MR spectroscopy ( $\left.{ }^{1} \mathrm{H}-\mathrm{MRS}\right)$, by analyzing pre- and postexercise difference spectra (4). Recently, we showed that long TE spectroscopy promotes the detectability and hence the quantification of the acetylcarnitine peak at $2.13 \mathrm{ppm}$ due to TE-induced suppression of overlapping lipid resonances, thereby enabling acetylcarnitine detection even in the absence of (exercise) interventions (5).

There is a relatively large difference in transversal relaxation time $\left(\mathrm{T}_{2}\right)$ between acetylcarnitine and lipid resonances, causing the lipid signal to decay rapidly with increasing TE. Increased suppression of the lipid signal can be achieved by increasing TE, but this goes at the expense of a lower signal-to-noise ratio (SNR) of the acetylcarnitine signal. With an echo time of $350 \mathrm{~ms}$, acetylcarnitine is generally well detectable in a large $(48 \mathrm{~mL})$ voxel in the upper leg (5). In obese subjects, with high myocellular lipid levels, a residual lipid signal can however still result in considerable contamination of the acetylcarnitine resonance. This interferes with accurate quantification of the acetylcarnitine concentration in subjects with high lipid content in muscle, even more so, as these subjects usually show low acetylcarnitine concentrations. Alternative sources of contrast might help to improve the suppression of lipid signals in these cases.

An obvious alternative to $\mathrm{T}_{2}$ relaxation based contrast is the use of spin-lattice relaxation time $\left(\mathrm{T}_{1}\right)$ weighing. It is known that the spin-lattice relaxation time of lipids is relatively short $(6,7)$ when compared with other metabolites, such as total creatine (t-Cr) (6) but also acetylcarnitine. The precise $\mathrm{T}_{1}$ of acetylcarnitine has not been reported, but we previously estimated the $\mathrm{T}_{1}$ of acetylcarnitine to be $2000 \mathrm{~ms}$ at 3T. This is also why we used a relatively long TR of $6000 \mathrm{~ms}$ (5) in our previous report, which was required to achieve sufficient SNR.

$\mathrm{T}_{1}$ contrast is traditionally achieved by using short TR. As short TR results in suppression of signals with a long $\mathrm{T}_{1}$ relaxation time (like acetylcarnitine), it is key to reverse this traditional $\mathrm{T}_{1}$ contrast for acetylcarnitine detection and for the concomitant suppression of the lipid resonances. This can be realized by alternating standard signal acquisition with an inversion recovery 

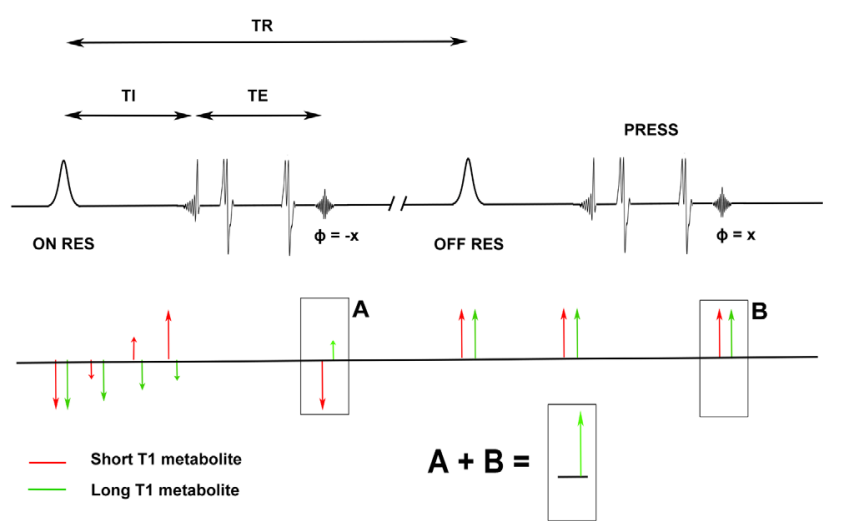

FIG. 1. Schematic representation of the protocol to achieve $T_{1}$ editing. Signal acquisition with an inversion recovery sequence, using PRESS localization, is alternated with regular signal acquisition by setting the resonance frequency of the second adiabatic inversion pulse far off resonance $(+50 \mathrm{kHz})$. The phase of the receiver is alternated concomitantly to suppress short $T_{1}$ metabolites (in red). Long $T_{1}$ metabolites are not relaxed to equilibrium when using intermediate TI times, leading to incomplete subtraction of these metabolites and thus observable signals.

sequence with an intermediate inversion time (TI). Subtraction of both signals will result in destruction of short $\mathrm{T}_{1}$ signals, while metabolites with a long $\mathrm{T}_{1}$ will be retained. We here explored the use of this approach for the detection of acetylcarnitine in skeletal muscle in vivo.

\section{METHODS}

\section{Methodology}

The approach used in this study is schematically depicted in Figure 1. Essentially, in the first acquisition an inversion recovery sequence is applied, using an adiabatic hyperbolic secant pulse with a bandwidth of $5000 \mathrm{~Hz}$ to invert all spins and a Point Resolved Spectroscopy (PRESS) (8) sequence for volume selection. In a second acquisition, the resonance frequency of the adiabatic inversion pulse is set far off resonance $(+50 \mathrm{kHz})$, leaving the magnetization on resonance unaffected and effectively reducing the sequence to a normal PRESS scheme.

We subtracted the noninverted spectrum from the spectrum acquired with the inversion pulse, by alternating the phase of the receiver. As metabolites with a short $\mathrm{T} 1$ relaxation time will recover faster to equilibrium during the first acquisition, their contribution to the overall signal is cancelled due to the subtraction scheme. Signal yield will be highest for metabolites with a long T1. As this approach is based on two separate acquisitions, overall signal intensity will be lower when compared with a nonedited acquisition within the same time frame.

In this setting, optimal contrast between two metabolites is dependent on their respective $\mathrm{T}_{1}$ relaxation times. To illustrate this, we have plotted the relative signal intensity (as $\% \mathrm{M}_{0}$ ) as a function of the $\mathrm{T}_{1}$ relaxation time of a metabolite for three different TI's (TI $=300$, 900, or $1500 \mathrm{~ms}$, see Figure 2). The essence of our approach, i.e., suppression of short $\mathrm{T}_{1}$ metabolites, is observable directly in this plot. Signal intensity increases with increasing $\mathrm{T}_{1}$. In the present case, we aim at near complete suppression of the lipid signals in the region of 2.2-2.4 ppm. These lipid resonances have $\mathrm{T}_{1}$ relaxation rates between 200 and $450 \mathrm{~ms}$ (7). For a TI of $900 \mathrm{~ms}$, at least $90 \%$ lipid suppression in this region is achieved, which comes with an approximate $40 \%$ signal loss for the acetylcarnitine resonance (or any resonance with a

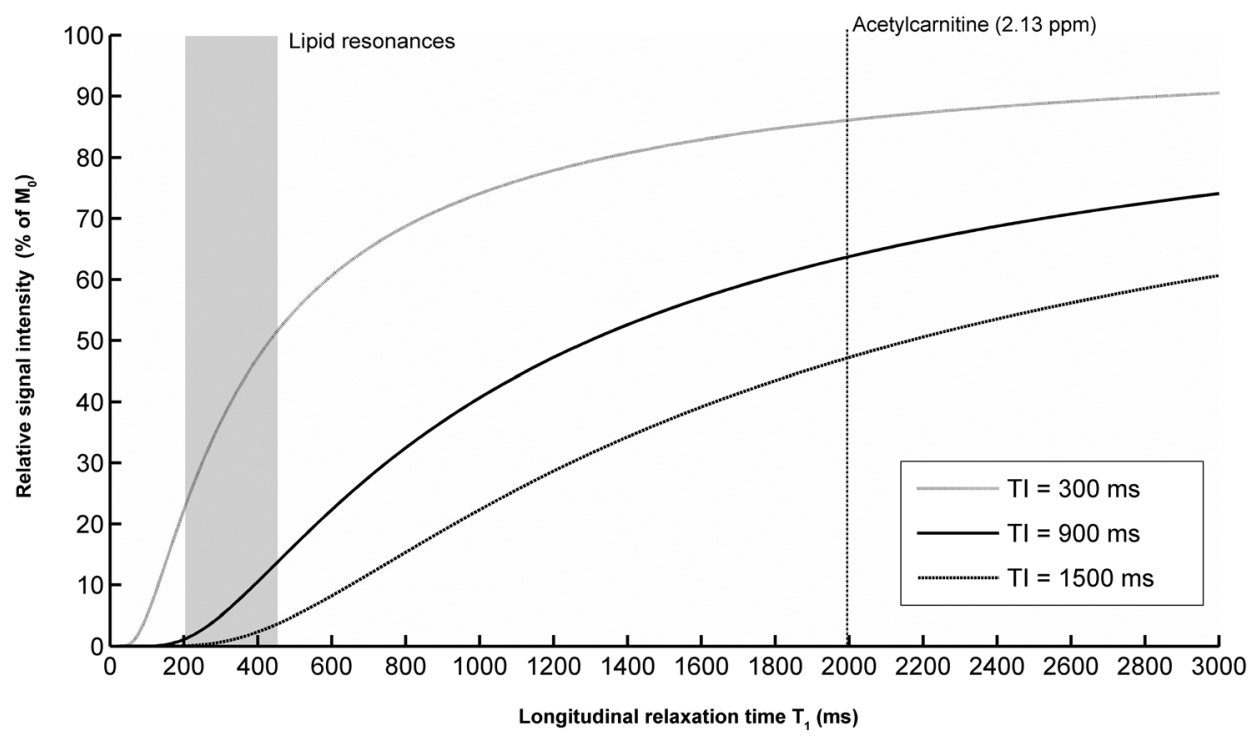

FIG. 2. Calculated relative signal intensity with the $T_{1}$ editing approach as a function of $T_{1}$ relaxation time, for three different $T I$ times. Signal intensity is given relative to equilibrium magnetization $\left(M_{0}\right)$. The lipid resonances are characterized by $T_{1}$ relaxation times between 200 and $450 \mathrm{~ms}$ (gray bar), while we estimated the acetylcarnitine $\mathrm{T}_{1}$ to be $2000 \mathrm{~ms}$ (dotted vertical line). Relative signal intensity is plotted for a TI of 300,900 , and $1500 \mathrm{~ms}$. To ensure a suppression of the lipid signals by a factor of 10 , a Tl of 900 ms was used in the in vivo protocol. Increasing $\mathrm{TI}$ will lead to increased suppression of short $\mathrm{T}_{1}$ metabolites, but decreased relative acetylcarnitine signal intensity. Coming with the increased lipid suppression at a TI of $900 \mathrm{~ms}$, the signal intensity of the acetylcarnitine will decay to be approximately $60 \%$ of $\mathrm{M}_{0}$. 
$\mathrm{T}_{1}$ of $2000 \mathrm{~ms}$ ). Increasing $\mathrm{TI}$ will lead to an improved suppression of short $\mathrm{T}_{1}$ metabolites, but this also comes with a decreased signal intensity of the signal of interest.

\section{Set-up and Subjects}

To test the performance of this novel approach, measurements were performed in a total of five subjects. In first instance, the sequence was evaluated in one a healthy subject [female, age 30 and body mass index BMI) $18 \mathrm{~kg} /$ $\mathrm{m}^{2}$ ], in whom acetylcarnitine concentration was relatively high and lipid content low. Next, the added value of the novel approach above the earlier reported long TE spectroscopy was tested in four overweight/obese subject (male subjects, age $68 \pm 3$ years and BMI $29 \pm 3 \mathrm{~kg} / \mathrm{m}^{2}$ ) with strong lipid signals masking the small acetylcarnitine peak. Experiments were approved by the institutional medical ethics committee and written informed consent was obtained from the subjects before these experiments.

\section{MRS Acquisition Protocol}

All experiments were performed on a 3 Tesla $(\mathrm{T})$ clinical MR system (Achieva 3T-X, Philips Healthcare, Best, The Netherlands) using a two-element flexible surface receive coil. Subjects were positioned supine and feet first in the magnet bore with the right foot constrained by two sandbags. The coil was placed over the vastus lateralis muscle. $\mathrm{T}_{2}$-weighted turbo spin echo images were acquired, consisting of three transversal slices and field of view $(\mathrm{FOV})=250 \times 210 \mathrm{~mm}$, slice thickness $=0.9 \mathrm{~mm}$, repetition time/echo time $(\mathrm{TR} / \mathrm{TE})=2000 / 100 \mathrm{~ms}$ and turbo factor 20 .

All spectra were acquired with a TR of $6000 \mathrm{~ms}$. A voxel of $40 \mathrm{~mm} \times 20 \mathrm{~mm} \times 60 \mathrm{~mm}$ was positioned in the vastus lateralis muscle. Outer volume suppression using three rest slabs was applied, to eliminate residual signals of subcutaneous adipose tissue. An example of voxel placement in the vastus lateralis muscle in one of the obese subjects is shown in Figure 4A. Spectral bandwidth was $2 \mathrm{kHz}$, number of acquired data points 2048, number of averages (NSA) 20 and a four-step phase cycling was applied. Shimming was performed with FASTMAP-based shimming (9).

As proof of principle in the lean subject, a short TE (40 ms) PRESS spectrum was acquired and compared with acquisition with the $\mathrm{T}_{1}$ editing approach with a TI of $900 \mathrm{~ms}$ and identical TE. Subsequently, a long TE (350 ms) PRESS spectrum without $\mathrm{T}_{1}$ editing was compared with the $\mathrm{T}_{1}$ editing approach using an intermediate and long TE (150 ms and $350 \mathrm{~ms}$ respectively). As water has a $\mathrm{T}_{1}$ of approximately $1400 \mathrm{~ms}$ (6), we implemented water suppression by selective excitations and crusher gradients just before the PRESS excitation pulse. The flip angle of the selective excitation pulse was empirically set at 110 degrees.

In one of the obese subjects, also spectra with short $\mathrm{TE}$, with and without $\mathrm{T}_{1}$ editing, were acquired for comparison. In all four obese subjects, we compared the long TE protocol with and without $\mathrm{T}_{1}$ editing (using $\mathrm{TE}$ of 150 and $350 \mathrm{~ms}$ ). Additionally, in a separate experiment in one of the obese subjects we intentionally placed the
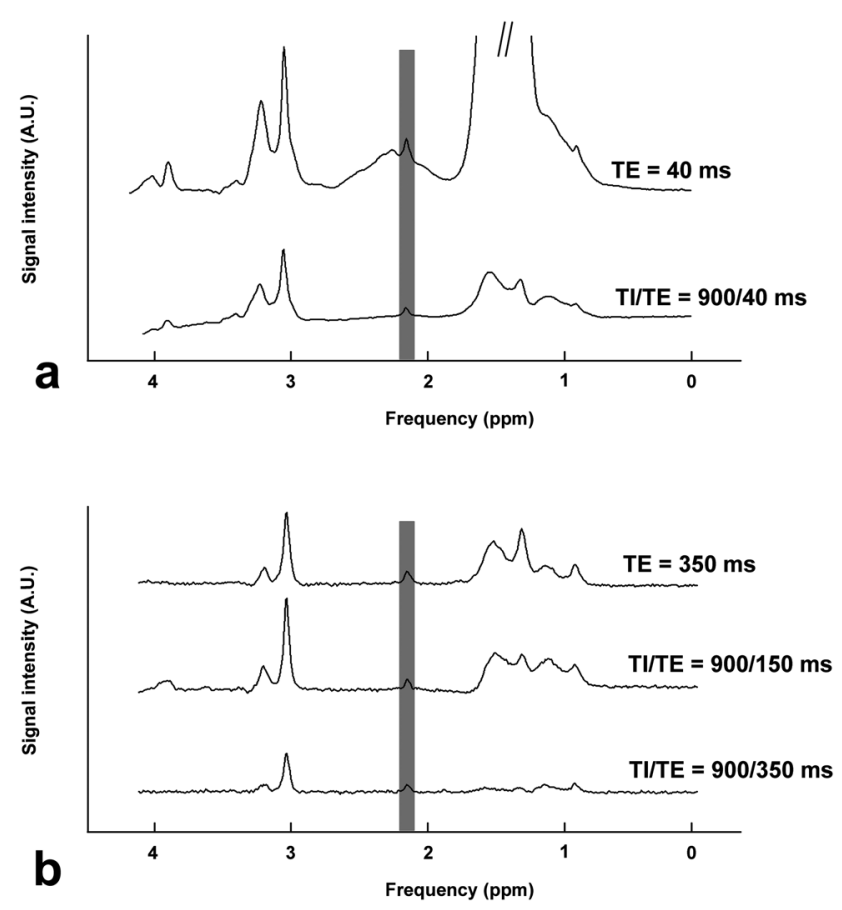

FIG. 3. Proof of principle experiment in a lean subject. Spectra are acquired from the vastus lateralis muscle in a lean subject. A: Acquisition of a regular PRESS spectrum with TE $=40 \mathrm{~ms}$ is compared with the $T_{1}$ editing approach with $T I / T E=900 / 40 \mathrm{~ms}$. Lipid signals are suppressed with $T_{1}$ editing. The acetylcarnitine peak at $2.13 \mathrm{ppm}$ is accentuated in the grey box. B: The long TE protocol is compared with the $T_{1}$ editing approach in the same subject. The $T_{1}$ editing approach $(T I=900 \mathrm{~ms})$ with a $T E$ of $150 \mathrm{~ms}$ leads to comparable acetylcarnitine and lipid peak intensity, when compared with the long TE $(350 \mathrm{~ms})$ protocol. Contrast between EMCL and IMCL is slightly different, suggesting shorter $T_{1}$ of the IMCL signal. Combination of the long TE protocol with the $T_{1}$ editing approach leads to almost complete suppression of the lipid signals.

voxel partially (approximately 10\% of the total voxel volume) in the subcutaneous adipose tissue layer to maximize lipid contamination. We here tested the $\mathrm{T}_{1}$ editing approach with a TE of $350 \mathrm{~ms}$ and compared this with a long TE spectrum alone. We here omitted the rest slabs for outer volume suppression.

\section{RESULTS}

\section{Proof of Principle in Lean Subject}

Using the $\mathrm{T}_{1}$ editing approach we were able to suppress the lipid signals in the region from 0.9 to $2.5 \mathrm{ppm}$ in the short TE spectrum of the lean subject, as is visible in Figure 3A. Upon application of the $\mathrm{T}_{1}$ editing approach, the acetylcarnitine peak at $2.13 \mathrm{ppm}$ is no longer masked by lipids and appears as a single peak.

A comparison between the previously described long TE protocol with and without $\mathrm{T}_{1}$ editing indicates that a TI of $900 \mathrm{~ms}$ in combination with a TE of $150 \mathrm{~ms}$ results in similar spectra, in terms of lipid suppression and acetylcarnitine peak intensity, as illustrated in Figure 3B. The combination of the long $\mathrm{TE}$ protocol and $\mathrm{T}_{1}$ editing shows the additive effect of the two approaches on lipid suppression. This results in a spectrum with the lowest 


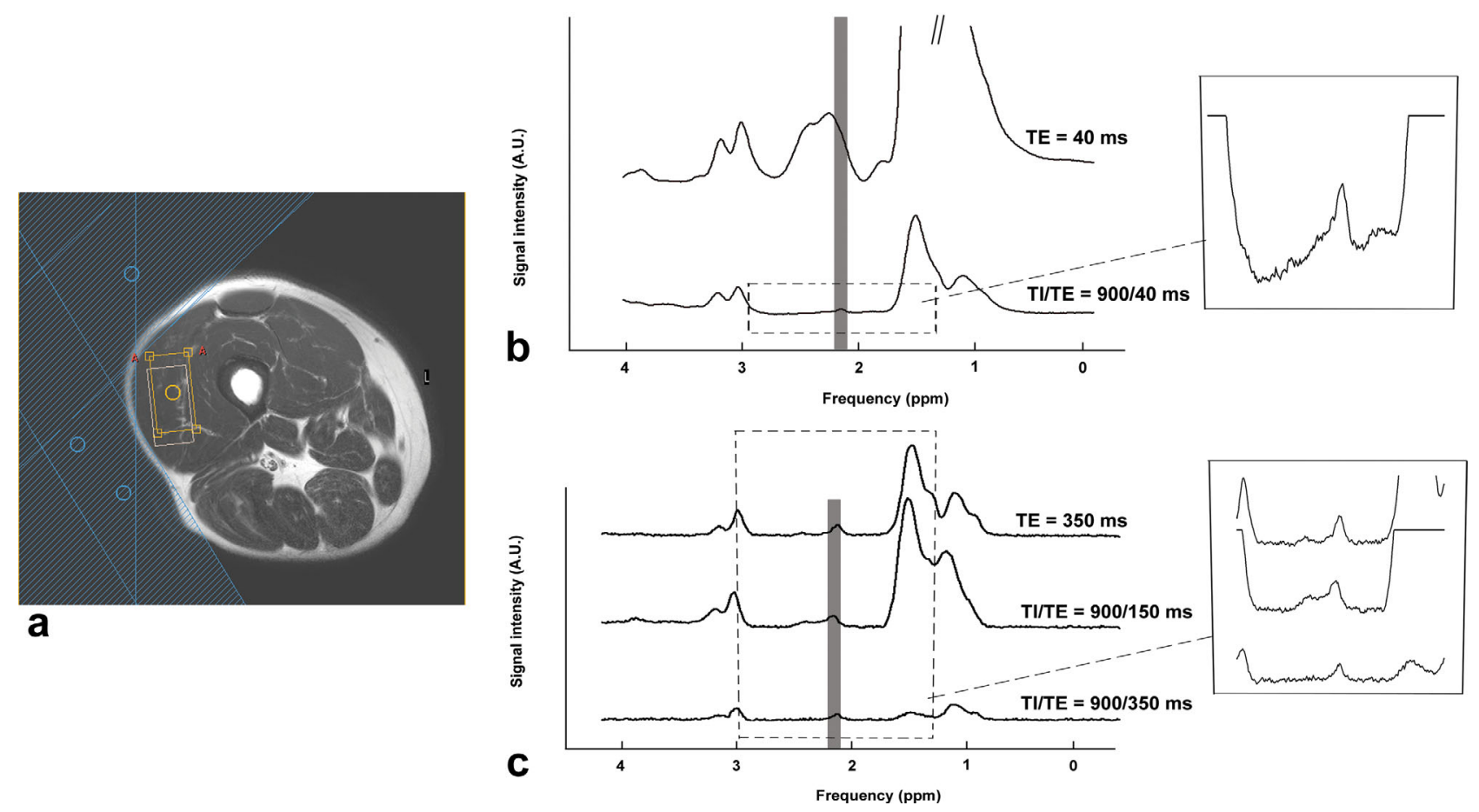

FIG. 4. Performance of the $T_{1}$ editing approach in an obese subject. A: The voxel placement in the vastus lateralis muscle is shown. Rest slabs for outer volume suppression are shown in blue. The chemical shift dependent voxel locations are shown for the lipid resonance at $1.2 \mathrm{ppm}$ (yellow box) and the $\mathrm{t}-\mathrm{Cr}$ resonance at $3.03 \mathrm{ppm}$ (white box). B: The results are depicted for short TE with and without $\mathrm{T}_{1}$ editing $(\mathrm{TE}=40$ versus $\mathrm{Tl} / \mathrm{TE}=900 / 40 \mathrm{~ms}$ ). A closer look on the acetylcarnitine region shows that residual lipid signals are still present with short $T E+T_{1}$ editing. In $\mathbf{C}, T_{1}$ editing is combined with longer TE. The acetylcarnitine region of the spectrum is shown in more detail on the right part of figure. A TE of $150 \mathrm{~ms}$ leads to insufficient suppression of the lipid signals. Identification and quantification of the acetylcarnitine peak is also hampered in the long $T E$ protocol alone ( $T E=350 \mathrm{~ms}$ ). A combination of the long $T E$ protocol and $T_{1}$ editing with TI 900 ms, leads to a well-resolved and well-defined acetylcarnitine peak. In panels B and C, the grey box is used to accentuate the acetylcarnitine peaks at $2.13 \mathrm{ppm}$.

remaining lipid signals, which comes with signal losses of approximately $50 \%$ on the t-Cr (at $3.03 \mathrm{ppm}$ ) and $40 \%$ on the acetylcarnitine peak.

\section{Application in Overweight/Obese Subjects}

In Figure $4 \mathrm{~B}$, the short $\mathrm{TE}$ spectra with and without $\mathrm{T}_{1}$ editing are shown for one of the obese subjects. In the short TE spectrum alone, large lipid signals are visible. When zooming in on the acetylcarnitine region it is clear that these lipid signals are significantly reduced with the $\mathrm{T}_{1}$ editing approach. However, residual lipid signals that are still present make it difficult to accurately phase the spectrum. As depicted for the same obese subject in Figure $4 \mathrm{C}$, we found that for all four obese subjects, both the long $\mathrm{TE}$ protocol and $\mathrm{T}_{1}$ editing approach with (intermediate) TE of $150 \mathrm{~ms}$, showed residual lipid peaks overlapping with the acetylcarnitine resonance, thereby hindering accurate quantification of this peak. Combination of the long TE protocol with the $\mathrm{T}_{1}$ editing approach with a TI of $900 \mathrm{~ms}$, resulted in enhanced suppression of lipid signals, which in turn results in a well-resolved acetylcarnitine peak in all subjects. In analogy with the lean subject, we estimated that the $\mathrm{T}_{1}$ editing approach comes with signal losses of approximately $50 \%$ for the tCr (at $3.03 \mathrm{ppm}$ ) and $40 \%$ for the acetylcarnitine (when assuming equal TE).

\section{Suppression of Adipose Tissue Contamination}

We have shown the spectra of the separate experiment, where we intentionally placed a part of the voxel in the subcutaneous adipose tissue, in Figure 5. In the long TE spectrum the lipid signals from the adipose tissue clearly contaminate the acetylcarnitine signal, which makes the peak unobservable. The combination of the long TE protocol and the $\mathrm{T}_{1}$ editing approach however result in complete suppression of the adipose tissue lipid signals, thereby uncovering the acetylcarnitine peak. Signal intensity of the acetylcarnitine peak was comparable with the signal intensity for the acetylcarnitine peak when the voxel was completely positioned inside the muscle tissue, which illustrates further that adipose tissue signals were completely suppressed in the acetylcarnitine region.

\section{DISCUSSION AND CONCLUSIONS}

We have shown here that differences in $\mathrm{T}_{1}$ relaxation times of the overlapping resonances of lipids and acetylcarnitine, offer an alternative contrast mechanism to the previously described $\mathrm{T}_{2}$ induced contrast (5) with long TE. Signal suppression of short $\mathrm{T}_{1}$ metabolites, e.g., lipids, can be achieved by subtraction of signals from an inversion recovery of intermediate TI times (in the order of $1000 \mathrm{~ms}$ ) from signals from regular signal acquisition. 
FIG. 5. Suppression of subcutaneous adipose tissue contamination with $T_{1}$ editing. Intentional placement of part of the voxel in the subcutaneous adipose tissue around the vastus lateralis muscle, leads to large lipid signals, even with $\mathrm{TE}=350 \mathrm{~ms}$. The acetylcarnitine peak cannot be resolved in this case. Lipid signals are suppressed by the use of the $T_{1}$ editing approach $(\mathrm{TI}=900 \mathrm{~ms})$, as is shown in more detail in the zoom view of the region of the acetylcarnitine resonance ( 1.5-3 ppm region).

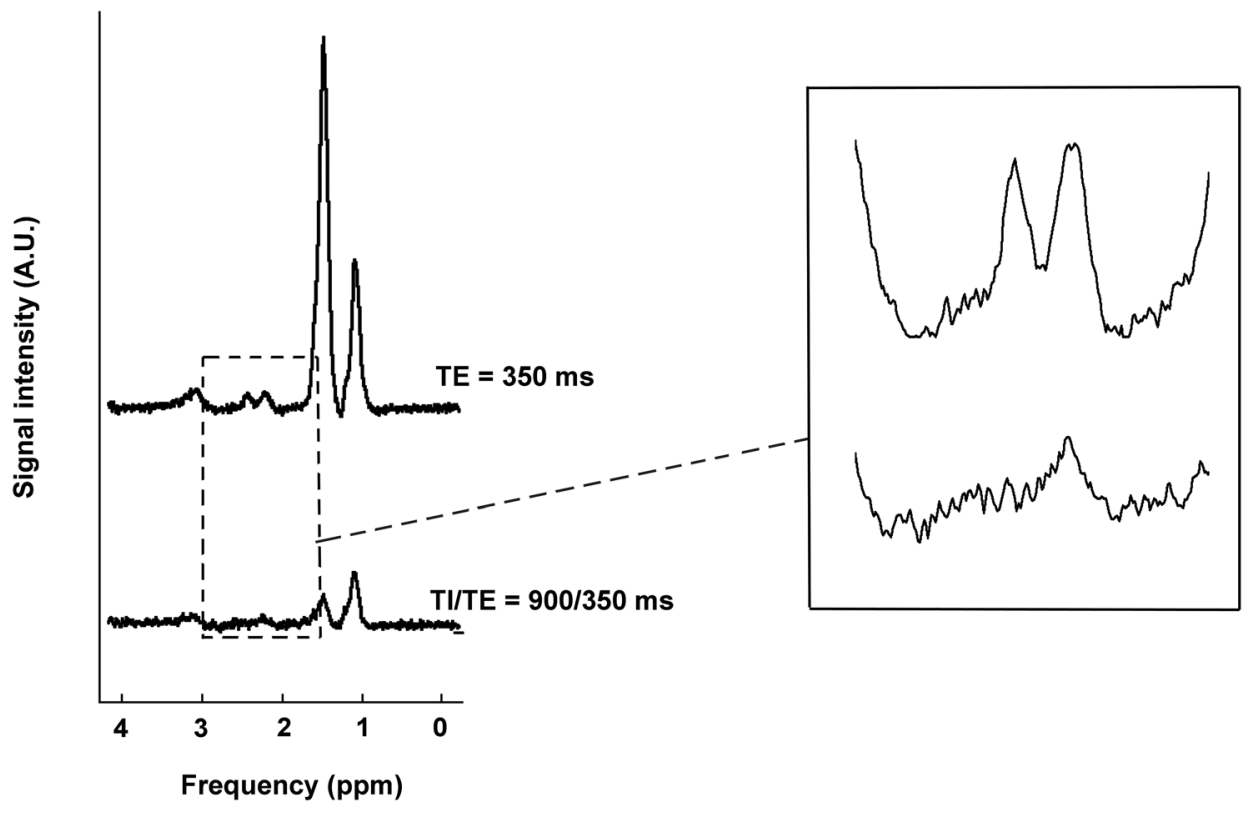

While this approach offers $\mathrm{T}_{1}$ contrast in itself, combination of the long TE protocol with the $\mathrm{T}_{1}$ editing approach offers improved lipid suppression and thus enhanced acetylcarnitine visibility and more accurate quantification in subjects with (very) high extra- and intramyocellular lipid signals.

The detection of acetylcarnitine in vivo, with ${ }^{1} \mathrm{H}-\mathrm{MRS}$ was first shown by subtraction of pre- and post-exercise spectra (4), which limited its use to studies in which an exercise intervention was performed. Recently, it was suggested that acetylcarnitine formation may play a crucial role in the capability of switching between basal fat oxidation and insulin-stimulated glucose oxidation in muscle (termed metabolic flexibility). Furthermore, the capacity to form acetylcarnitine might be hampered in insulin resistant subjects $(1,2,5)$. To understand the role of acetylcarnitine in more detail, it is crucial to develop MR acquisition strategies that enable detection of this metabolite at rest, without the need for an (exercise) intervention. The relatively short $\mathrm{T}_{2}$ of lipids enables to suppress these signals by prolonging the TE in a regular PRESS sequence (5). For a TE of $350 \mathrm{~ms}$, one can calculate that approximately $98 \%$ of the lipid signal is suppressed at $3 \mathrm{~T}$, which is sufficient for a large subset of subjects. For obese subjects, with high myocellular lipid content, the suppression might, however, not suffice for accurate quantification of the acetylcarnitine signal. Augmenting lipid suppression by increasing TE above 350 ms is possible, but will unavoidably also lead to unwanted loss of acetylcarnitine signal and hence long acquisition times.

In a strive to optimize acetylcarnitine quantification for subjects with high myocellular lipid content, we estimated that differences in relative $\mathrm{T}_{1}$ relaxation times of lipids and acetylcarnitine are even more pronounced than differences in $\mathrm{T}_{2}$. These differences in $\mathrm{T}_{1}$ relaxation times are exploited here. As in theory, the $\mathrm{T}_{1}$ editing approach with short TE gives better contrast-to-noise ratio, or improved lipid suppression at same acetylcarni- tine signal intensities, when compared with the long TE protocol, we anticipated the $\mathrm{T}_{1}$ editing approach to be superior to the long TE protocol. In practice however, system stability was limiting complete suppression of the lipid signals due to subtraction artifacts with the $\mathrm{T}_{1}$ editing approach. Therefore, the single shot, long TE protocol, and subtraction-based $\mathrm{T}_{1}$ editing protocol performed very similarly.

As an alternative strategy to use $\mathrm{T}_{1}$ differences between acetylcarnitine and lipids, the conventional inversion recovery sequence might also be used for nulling of the lipid resonances that cover the acetylcarnitine resonance. While this approach is more sensitive to variations in $\mathrm{T}_{1}$ relaxation times, the absence of a subtraction scheme and a higher signal intensity $\left(\sim 80 \%\right.$ of $\mathrm{M}_{0}$ with IR with $\mathrm{TI}=200 \mathrm{~ms}$ vs. $\sim 60 \%$ of $\mathrm{M}_{0}$ with the proposed $\mathrm{T}_{1}$ editing approach) are advantageous. Initial experiments using this approach, however, resulted in spectra that could be not be accurately phased in the acetylcarnitine region. This can be explained by the fact that in 2.2-2.4 ppm region two separate lipid resonances are contaminating the acetylcarnitine signal and these lipid resonances are characterized by different $\mathrm{T}_{1}$ relaxation times (7). Nulling of the $2.2 \mathrm{ppm}$ resonance on one hand will lead to a negative signal component from the $2.4 \mathrm{ppm}$ resonance. In contrast, with the proposed $\mathrm{T}_{1}$ editing approach the degree of suppression increases with decreasing $\mathrm{T}_{1}$ relaxation times for a given TI. Nulling of the $2.4 \mathrm{ppm}$ resonance automatically also suppresses the shorter $\mathrm{T}_{1}$ resonance at $2.2 \mathrm{ppm}$.

For the lean subject, with low myocellular lipid signals, lipid suppression and accurate acetylcarnitine quantification could already be achieved with the $\mathrm{T}_{1}$ editing approach with a TI of $900 \mathrm{~ms}$ and with short TE of $40 \mathrm{~ms}$. Importantly, the $\mathrm{T}_{1}$ editing approach can also be combined with the long TE protocol to maximize the suppression of lipid resonances. As both low acetylcarnitine concentrations and high lipid contamination are characteristic for obese subjects and patients with type 2 
diabetes (T2DM), the use of the $\mathrm{T}_{1}$ editing approach in combination with the long TE protocol leads to enhanced suppression of the lipid signals covering the (small) acetylcarnitine peak in these subjects, enabling more accurate quantification of this peak. The power of this approach is also illustrated by a separate experiment in one of the obese subjects where we intentionally placed the voxel partially in the subcutaneous adipose tissue layer to maximize lipid contamination. The $\mathrm{T}_{1}$ editing approach with long TE (350 ms) still resulted in complete suppression of the adipose tissue lipid signals and resulted in a well resolved acetylcarnitine peak. This peak was completely covered by lipid resonances in the long TE protocol alone.

Of course, not only the lipid and acetylcarnitine signal intensities are affected by the proposed $\mathrm{T}_{1}$ editing approach. As can be deduced from the spectra acquired with this approach, the t-Cr peak is also well preserved in the spectra. This peak can be used as an internal reference for absolute quantification of acetylcarnitine. In our experiments we estimated the signal losses on approximately $50 \%$ for t-Cr and $40 \%$ for acetylcarnitine, which is well in line with the theoretically estimated signal loss (based on a T1 of 1100 (6) ms for t-Cr and $2000 \mathrm{~ms}$ for acetylcarnitine, see also Figure 2). Thus, for absolute quantification, additional corrections should be applied to compensate for the $\mathrm{T}_{1}$ induced signal losses on all the metabolites of interest. Accurate determination of the $\mathrm{T}_{1}$ relaxation times of $\mathrm{t}-\mathrm{Cr}$ and acetylcarnitine is warranted in the target patient group.

However, as acetylcarnitine concentrations are changing rapidly, care has to be taken to account for these fluctuations during the determination of relaxation times. Also, changes in $\mathrm{T}_{1}$ and $\mathrm{T}_{2}$ relaxation times with for example disease or after exercise, might induce quantifications errors or bias when comparing different groups. While exact data on the $\mathrm{T}_{1}$ relaxation time of acetylcarnitine are lacking, it can be estimated that when the proposed $\mathrm{T}_{1}$ editing approach is used, a $\mathrm{T}_{1}$ difference of $-350 \mathrm{~ms}$ (e.g., $\mathrm{T}_{1}=1650 \mathrm{~ms}$ instead of $2000 \mathrm{~ms}$ ) would lead to an underestimation of $10 \%$ of the acetylcarnitine concentration. A $\mathrm{T}_{1}$ difference of $+500 \mathrm{~ms}$ (e.g., $\mathrm{T}_{1}=2500 \mathrm{~ms}$ instead of $2000 \mathrm{~ms}$ ) would lead to an overestimation of around $10 \%$. Thus, although the approach leads to heavy $\mathrm{T}_{1}$ weighing, the intrinsic long $\mathrm{T}_{1}$ of acetylcarnitine reduces the risk for quantification errors with changing $\mathrm{T}_{1}$ rates. For $\mathrm{T}_{2}$, either a positive or negative shift of $20 \mathrm{~ms}$ would lead to under- or overestima- tion of $10 \%$. Importantly, we have previously shown that the $\mathrm{T}_{2}$ relaxation time for acetylcarnitine is similar for lean and obese subjects (5). Furthermore, $\mathrm{T}_{2}$ of acetylcarnitine did not differ pre- and postexercise in lean subjects (data not published).

Overall, we conclude that the $\mathrm{T}_{1}$ editing approach enhances the visibility of overlapping metabolites with a long $\mathrm{T}_{1}$. Using $\mathrm{T}_{1}$ editing in addition to the long TE protocol is a feasible strategy to improve detection of the acetylcarnitine peak in subjects with high lipid signals in skeletal muscle, although signal intensity is reduced by approximately $40 \%$ when compared with the long TE protocol alone. The combination of $\mathrm{T}_{1}$ editing with long $\mathrm{TE}$ optimally suppresses lipid signals and achieves nearcomplete cancellation of lipid signals, even in obese subjects where myocellular lipids are abundant. Importantly, as acetylcarnitine metabolism seems to be disturbed in obesity and T2DM, these patients are the most interesting population to study in that respect. The proposed technique will hence be essential to advance the study on the significance of carnitine metabolism in obesity and T2DM. The application of the $\mathrm{T}_{1}$ editing approach in other tissues, and in identifying metabolites that have been uncovered to date, requires future evaluation.

\section{REFERENCES}

1. Muoio DM, Noland RC, Kovalik JP, et al. Muscle-specific deletion of carnitine acetyltransferase compromises glucose tolerance and metabolic flexibility. Cell Metab 2012;15:764-777.

2. Noland RC, Koves TR, Seiler SE, Lum H, Lust RM, Ilkayeva O, Stevens RD, Hegardt FG, Muoio DM. Carnitine insufficiency caused by aging and overnutrition compromises mitochondrial performance and metabolic control. J Biol Chem 2009;284:22840-22852.

3. Muoio DM. Metabolic inflexibility: when mitochondrial indecision leads to metabolic gridlock. Cell 2014;159:1253-1262.

4. Kreis R, Jung B, Rotman S, Slotboom J, Boesch C. Non-invasive observation of acetyl-group buffering by $1 \mathrm{H}-\mathrm{MR}$ spectroscopy in exercising human muscle. NMR Biomed 1999;12:471-476.

5. Lindeboom L, Nabuurs CI, Hoeks J, et al. Long-echo time MR spectroscopy for skeletal muscle acetylcarnitine detection. J Clin Invest 2014;124:4915-4925.

6. Krssak M, Mlynarik V, Meyerspeer M, Moser E, Roden M. 1H NMR relaxation times of skeletal muscle metabolites at 3 T. MAGMA 2004; 16:155-159.

7. Wang L, Salibi N, Wu Y, Schweitzer ME, Regatte RR. Relaxation times of skeletal muscle metabolites at 7T. J Magn Reson Imaging 2009;29:1457-1464.

8. Bottomley PA. Spatial localization in NMR spectroscopy in vivo. Ann N Y Acad Sci 1987;508:333-348.

9. Gruetter R. Automatic, localized in vivo adjustment of all first- and second-order shim coils. Magn Reson Med 1993;29:804-811. 